\title{
On the Classical Energy Equipartition Theorem
}

\author{
J. A. S. Lima ${ }^{1 *}$ and A. R. Plastino ${ }^{2 \dagger}$ \\ ${ }^{1}$ Universidade Federal do Rio Grande do Norte, \\ Departamento de Física, Caixa Postal 1641, \\ 59072-970 Natal, RN, Brazil \\ ${ }^{2}$ Facultad de Ciencias Astronomicas y Geofisicas \\ Universidad Nacional de La Plata, C.C. 727, 1900 La Plata, Argentina \\ Received 20 April 1999. Revised version received on 17 September 1999
}

\begin{abstract}
A general proof of the energy equipartition theorem is given. Our derivation holds for any distribution function depending on the phase space variables only through the Hamiltonian of the system. This approach generalizes the standard theorem in two main directions. On the one hand, it considers the contribution to the total mean energy of homogeneous functions having a more general type than the ones usually discussed in the literature. On the other hand, our proof does not rely on the assumption of a Boltzmann-Gibbs exponential distribution.
\end{abstract}

The classical energy equipartition theorem constitutes an important result in thermodynamics, statistical mechanics and kinetic theory, which has been extensively discussed in the literature [1-9]. Its simplest version deals with the contribution to the mean energy of a system in thermal equilibrium at temperature $T$ due to quadratic terms in the Hamiltonian. More precisely, it attests that any canonical variable $x$ entering the Hamiltonian through an additive term proportional to $x^{2}$ has a thermal mean energy equal to $\frac{k T}{2}$, where $\mathrm{k}$ is the Boltzmann constant. The most familiar example is provided by a three dimensional classical ideal gas with Hamiltonian $\sum_{i} \mathbf{p}_{i}^{2} / 2 m$. The mean value of the kinetic energy associated with the $x$-component of the velocity of each particle is (ensemble average is denoted by \langle\rangle$)$,

$$
\left\langle\frac{1}{2} m \dot{x}^{2}\right\rangle=\left\langle\frac{1}{2 m} p_{x}^{2}\right\rangle=\frac{1}{2} k T
$$

Some time ago, the derivation of the equipartition theorem was generalized by Turner[7] in order to include the presence of nonquadratic terms. More precisely, assuming that the Hamiltonian $H\left(q_{i}, p_{i}\right), i=$ $1,2, \ldots f$, may be additively separated in the form

$$
H=g(x)+h,
$$

where $x$ denotes one of the $2 f$ coordinates or momenta, $g$ is an homogeneous function of degree $r$, and the function $h$ is independent of $x$, he shown that the mean

\footnotetext{
*limajas@dfte.ufrn.br

${ }^{\dagger}$ plastino@fcaglp.fcaglp.unlp.edu.ar
}

value of $g$ is given by

$$
\langle g\rangle=\frac{1}{r} k T .
$$

We emphasize that the proof furnished by Turner is valid only for one homogeneous degree of freedom and makes explicit use of the exponential Boltzmann-Gibbs distribution. In brief, the main goal of the present work is just to relax these two hypotheses. We believe that the analysis of the basic conditions needed for the validity of the equipartition theorem will contribute to the understanding of its physical meaning and to the clarification of its relationship with other physical principles and theorems. As it appears, the version of equipartition theorem presented here have at least two interesting virtues. The first one is to extend the scope of the theorem so as to incorporate the case of an homogeneous component of the Hamiltonian depending on many canonical variables. The second one is to demonstrate that the theorem is not an exclusive property of the exponential Boltzmann-Gibbs distribution function. We shall see that the theorem holds true essentially for any distribution function that depends on the canonical variables only through the system's Hamiltonian. As it is well known, this set of distribution functions constitutes a family of legitimate stationary solutions to Liouville's equation [3]. That is to say, they are fully consistent with the general principles of dynamics. As we point out below, these facts may also be exploited to good methodological advantage in presenting the equipartition theorem. 
Let us consider a Hamiltonian system with $f$ degrees of freedom (i.e., with a $2 f$-dimensional phase space) whose Hamiltonian function is of the form

$$
H=g\left(x_{1}, \ldots, x_{L}\right)+h,
$$

where $\left(x_{1}, \ldots, x_{L}\right)$ constitutes a subset of the $2 f$ phase space coordinates, $h$ does not depend on any of those $L$ variables, and $g$ is an homogeneous function of degree $r$. The homogeneity character of $g$ means that for any $\lambda>0$,

$$
g\left(\lambda x_{1}, \ldots, \lambda x_{L}\right)=\lambda^{r} g\left(x_{1}, \ldots, x_{L}\right) .
$$

What we are going to prove is that the only properties of $g$ determining its contribution $\langle g\rangle$ to the mean value of the Hamiltonian are its degree $r$ of homogeneity and the number $L$ of its arguments. Thus, our general form of the energy equipartition theorem can be stated as follows: All the homogeneous terms of the Hamiltonian that are characterized by the same values of the parameters $r$ and $L$ make the same contribution to the total mean energy. As already mentioned, we shall assume that our Hamiltonian system is described by a phase space probability density $f\left(q_{i}, p_{i}\right)$ depending on the canonical variables only through the system's Hamiltonian,

$$
f\left(q_{i}, p_{i}\right)=F\left[-\alpha-\beta H\left(q_{i}, p_{i}\right)\right],
$$

where $\alpha$ is a dimensionless constant determined by the normalization condition and the constant $\beta$ is such that the argument of the function $F$ is dimensionless. For instance, in the case of Gibbs' canonical ensemble we have $F(x)=e^{x}, \alpha$ is equal to the logarithm of the partition function $Z$, and the constant $\beta$ can be identified with the inverse temperature $1 / k T$.

Now, in order to compute the mean value of $g$, it proves convenient to introduce the function

$$
I(\lambda)=\int A\left[-\alpha-\beta\left\{g\left(\lambda x_{1}, \ldots, \lambda x_{L}\right)+h\right\}\right] d \Omega,
$$

where $d \Omega=d q_{1}, \ldots, d p_{f}$ is the phase space volume element and the function $A(x)$ is a primitive function of $F(x)$, that is

$$
A^{\prime}(x)=F(x) .
$$

By recourse to the change of integration variables

$$
x_{i}^{\prime}=\lambda x_{i}, \quad(i=1, \ldots, L),
$$

it is easy to see that

$$
I(\lambda)=\lambda^{-L} I_{1}
$$

where $I_{1}=I(\lambda=1)$. On the other hand, making explicit use of the homogeneity of $g$ one finds

$$
I(\lambda)=\int A\left[-\alpha-\beta\left\{\lambda^{r} g\left(x_{1}, \ldots, x_{L}\right)+h\right\}\right] d \Omega .
$$

Computing the logarithmic derivatives of expressions (10) and (11) and evaluating them at $\lambda=1$, we obtain, respectively,

$$
\left[\frac{1}{I} \frac{d I}{d \lambda}\right]_{\lambda=1}=-L
$$

and

$$
\left[\frac{1}{I} \frac{d I}{d \lambda}\right]_{\lambda=1}=-\frac{\beta r}{I_{1}}\langle g\rangle .
$$

These last two relations give us a simple expression for the mean value of $g$, namely

$$
\langle g\rangle=\frac{L}{r \beta} I_{1},
$$

or equivalently, by identifying $\beta$ with the inverse temperature $1 / k T$,

$$
\langle g\rangle=\frac{L}{r} k T I_{1} .
$$

This expression constitutes the main result of the present note. Before discussing the physical meaning of equation (15) a few comments on its mathematical derivation are in order. First of all, we have assumed that both the integrals defining the quantities $I$ (that is, equation (11) and $d I / d \lambda$ converge. That is to say, equation (15) is verified provided that the integrals for $I$ and $d I / d \lambda$ exist. The convergence of these integrals must be checked in each specific case by recourse to the standard techniques of the Calculus. This is just the usual situation with general theorems in theoretical physics, as can be illustrated by the following elementary example. Given a one dimensional, normalized probability distribution $p(x)$ it is well-known that

$$
\left\langle(x-\langle x\rangle)^{2}\right\rangle=\left\langle x^{2}\right\rangle-\langle x\rangle^{2} .
$$

However, this relation makes sense only if the integral $\left\langle x^{2}\right\rangle=\int x^{2} p(x) d x$ converges. There are important probability distributions for which the moment $\left\langle x^{2}\right\rangle$ diverges. As a famous example we have the Cauchy distribution

$$
p(x)=\frac{\pi^{-1}}{1+x^{2}} .
$$

In spite of these problematic cases there can be no doubt that it is both interesting and useful to know the general theorem (16), even if it holds only when the relevant integrals converge. Another moral that we can extract from the above little example is that it is not enough to restrict our considerations to the moments of positive quantities in order to avoid divergence difficulties. It should be stressed that in the case of the equipartition theorem the problem of convergence is by 
no means a consequence of our generalization. These kind of difficulties may arise also within the standard case of the Boltzmann-Gibbs statistical mechanics. For instance, in the case of a classical self gravitating $N$ body system not even the integral defining the partition function converges. Secondly, let us consider the limits of integration for the integral (11) and the associated integral for $d I / d \lambda$. If those limits go to $\pm \infty$ in all the variables $x_{1}, \ldots x_{L}$ then our derivation is clearly correct. Let us consider the case when those limits do not go to $\pm \infty$. In principle, this may happen by two different reasons:

- (1) The system has intrinsic constraints. For instance, if we have a particle moving inside the one dimensional box $[-L, L]$ it is plain that the limits of integration for the coordinate $x$ do not go to $\pm \infty$.

- (2) The limits of integration do not go to $\pm \infty$ because the distribution function $f\left(q_{i}, p_{i}\right)$ given by (6) has a cut-off (as happens, for example, with Tsallis distribution).

Our generalized version of the equipartition theorem does not hold in the case (1), and the same happens with the standard proof of the theorem. The equipartition theorem (as applied to the mean value of an additive term in the potential function) is not verified under this condition because a system endowed with those kind of constraints is, strictly speaking, outside the hypothesis of the theorem. Those constraints are described by "rigid wall" terms in the potential function associated with the system's Hamiltonian. The equipartition theorem is not applicable because potential functions exhibiting rigid walls are not, in general, homogeneous functions of the coordinates. It is clear that our generalization must not be blamed for this difficulty.

On the other hand, our theorem still holds true in the case (2). Let us assume that the function $F(x)$ appearing in the definition (6) of the phase space distribution function vanishes outside the interval $\left[a_{1}, a_{2}\right]$, but is continuous at the points $a_{1,2}$. We can then choose $A(x)$ (see equation (8)) in such a way that it vanishes too outside that interval, while being itself continuous and endowed with continuous derivatives at the points $a_{1,2}$. The boundary of the integration region associated with $I(\lambda)$ and $d I / d \lambda$ is then given by the two phase space hipersurfaces $\Sigma(\lambda)_{1,2}$ defined by the equations

$$
-\alpha-\beta\left\{g\left(\lambda x_{1}, \ldots, \lambda x_{L}\right)+h\right\}=a_{1,2} .
$$

When we perform the change (9) of integration variables it is easy to realize, according to elementary calculus, that in terms of the new variables $x_{i}^{\prime}$ the boundary of the integration region is now given by the equations

$$
-\alpha-\beta\left\{g\left(x_{1}^{\prime}, \ldots, x_{L}^{\prime}\right)+h\right\}=a_{1,2},
$$

and equation (10) follows immediately. As a final comment, notice that when one evaluates the derivative with respect to $\lambda$ of the expression $(7)$ for $I(\lambda)$, a surface term appears due to the dependence on $\lambda$ of the boundary of integration. This term, however, makes no contribution to $d I / d \lambda$ because the integrand $A$ vanishes at the integration boundary. Taking into account the above remarks and caveats, equations (12) and (13) are easily verified.

Equation (15) generalizes the standard energy equipartition theorem. For a system in equilibrium at a given temperature, it means that the contribution to the mean energy due to an homogeneous component $g$ of the Hamiltonian is only a function of the temperature $T$, the degree of homogeneity $r$, and the total number $L$ of arguments entering the function $g$. The mean value of $g$ does not depend upon its detailed structure. In particular, each quadratic term in the Hamiltonian depending on a single canonical variable (for example, each component of the kinetic energy) makes the same contribution to the total mean energy of the system, i.e.,

$$
\frac{1}{2} k T I_{1}
$$

It is important to stress that the expression (15) was obtained without any assumption about the specific form of the phase space distribution function. However, our general result does depend on the distribution function through the quantity (see $(7)$ )

$$
I_{1}=\int A(-\alpha-\beta H) d \Omega .
$$

In the case of Gibbs canonical ensemble, the distribution function adopts the form

$$
f\left(q_{i}, p_{i}\right)=\exp \left(-\alpha-\beta H\left(q_{i}, p_{i}\right)\right),
$$

where the parameter $\alpha$ is equal to the logarithm of the partition function $Z$ and has a value such that the distribution $f\left(q_{i}, p_{i}\right)$ is appropriately normalized, that is

$$
\int f\left(q_{i}, p_{i}\right) d \Omega=\int \exp \left(-\alpha-\beta H\left(q_{i}, p_{i}\right)\right) d \Omega=1
$$

Hence, we have $F(x)=\exp (x)$ and, since the only requirement on the function $A(x)$ is to be a primitive function of $F(x)$, we can take

$$
A(x)=F(x)=e^{x} .
$$

Thus, because of the normalization of the canonical distribution,

$$
I_{1}=\int F(-\alpha-\beta H) d \Omega=1 .
$$


Hence, within the Boltzmann-Gibbs formalism, our generalized theorem (15) leads to

$$
\langle g\rangle=\frac{L}{r} k T .
$$

Turner's result reduces to the particular $L=1$ case of the above expression, which describes the mean value of an homogeneous component of the Hamiltonian depending on one single canonical variable [7]. On the other hand, if $g$ is an homogeneous quadratic function, like the kinetic energy of the $N$ particles in a nonrelativistic classical gas ( $L=3 N, r=2$ ), we recover the standard version of the energy equipartition theorem usually appearing in textbooks of statistical mechanics[1-5]

$$
\langle g\rangle=\frac{3}{2} N k T .
$$

Notice, however, that the relation (26) is more general than the one usually found in textbooks, since it deals with homogeneous functions of arbitrary degree of homogeneity and arbitrary number of arguments. For instance, consider the case where the potential function depends on two generalized coordinates $q_{1}$ and $q_{2}$ through a term of the form

$$
g\left(q_{1}, q_{2}\right)=q_{1}^{2} q_{2} .
$$

This is an homogeneous function of degree 3 in the variables $q_{1}$ and $q_{2}$ and, consequently, we have $r=3$ and $L=2$. It thus follows that the mean value of $g$ evaluated on the Gibbs canonical ensemble is

$$
\langle g\rangle=\frac{2}{3} k T .
$$

Now we are going to illustrate the application of the general equipartition theorem to a nonexponential phase space distribution. Among the several available possibilities, let us consider the case of a distribution function exhibiting a power law dependence on the Hamiltonian. These kind of distribution functions appear naturally in many physical scenarios and are nowadays being intensively studied (see $[10,11]$ and references therein). For example, these power-law models have been successfully applied to describe the observed velocity distribution of clusters of galaxies [12]. The one-particle power-law distribution is

$$
f(H)=\frac{1}{Z_{q}}\left[1-(q-1) \beta \frac{1}{2} m \mathbf{v}^{2}\right]^{1 /(q-1)},
$$

where

$$
Z_{q}=\int\left[1-(q-1) \beta \frac{1}{2} m \mathbf{v}^{2}\right]^{1 /(q-1)} d^{3} v,
$$

and both $f$ and the integrand appearing in the definition of $Z_{q}$ are set equal to zero whenever the quantity between brackets becomes negative. We shall refer to this last requirement as the "cut-off" condition.

In this context, the functions $F(x)$ and $A(x)$ previously introduced now assume, respectively, the forms below

$$
F(x)=\frac{1}{Z_{q}}[1+(q-1) x]^{1 /(q-1)},
$$

and

$$
A(x)=\frac{1}{q Z_{q}}[1+(q-1) x]^{q /(q-1)} .
$$

The contribution of one velocity component to the mean kinetic energy is

$$
\left\langle\frac{1}{2} m v_{x}^{2}\right\rangle=\frac{1}{2} k T I_{1}
$$

with

$$
I_{1}=\frac{1}{q Z_{q}} \int\left[1-(q-1) \beta \frac{1}{2} m \mathbf{v}^{2}\right]^{q /(q-1)} d^{3} v .
$$

By taking $q>1$, and making the change of variables defined by

$$
\mathbf{u}=\left[(q-1) \beta^{\frac{1}{2}} m\right]^{-1 / 2} \mathbf{v},
$$

one may cheek that $I_{1}$ can be put under the guise

$$
I_{1}=\frac{1}{q} \frac{C_{1}}{C_{2}},
$$

where

$$
C_{1}=\int_{0}^{1} u^{2}\left[1-u^{2}\right]^{q /(q-1)} d u
$$

and

$$
C_{2}=\int_{0}^{1} u^{2}\left[1-u^{2}\right]^{1 /(q-1)} d u .
$$

The above two integrals are tabulated (see, for instance, [13]). Inserting their values into (37) one obtains

$$
I_{1}=\frac{2}{5 q-3},
$$

and from equation (34),

$$
\left\langle\frac{1}{2} m v_{x}^{2}\right\rangle=\frac{k T}{5 q-3} .
$$

Although evaluating $I_{1}$ under assumption $q>1$, it is not hard to prove that the expression (40) still holds true if $3 / 5<q \leq 1$. For $q$ values equal or lower than the critical value $q_{c}=3 / 5$, the mean values of $v_{x}^{2}$ and $v^{2}$ diverge. For $q<1 / 3$, nor even the probability distribution itself is normalizable. Like in the standard Boltzmann-Gibbs approach, it should be noticed that 
the quantity $I_{1}$ in this example does not depend on the temperature $T$. It is also worth remarking that, in the case of Tsallis statistics, we have illustrated our general theorem with the mean value of the kinetic energy which is always an extensive quantity. That is, the total kinetic energy of a classical $N$-body system is equal to the sum of the contributions due to each component of the velocities of each of its particles, even if the system exhibits an overall nonextensive behaviour due to long range interactions.

Naturally, as we have already mentioned, the general energy equipartition theorem (15) is meaningful only if the distribution function $f=F(-\alpha-\beta H)$ is normalizable and the integrals defining $\langle g\rangle$ and $I_{1}$ converge. Otherwise, relation (15) may lead to somewhat contradictory results. For example, in the case of a function $g$ endowed with a negative degree of homogeneity, relation (15) seems to imply that the mean value of $g$ must be negative, even if $g$ itself is always positive! This difficulty has already been pointed out by Turner[7]. In this concern, we remark that the distribution function $F(-\alpha-\beta H)$ is in general not normalizable when the Hamiltonian function has a term with a negative degree of homogeneity.

Summing up, we have shown that Hamiltonian dynamical systems decribed by phase space distributions depending only on the Hamiltonian comply with the strictures of a generalized energy equipartition theorem. Homogeneous terms in the Hamiltonian exhibiting the same degree of homogeneity and depending on the same number of canonical variables give the same contribution to the total mean energy. In the case of Gibbs canonical ensemble our version of the theorem reproduces the standard well-known results. However, it can also be applied within more general frameworks. Hopefully, the simple generalization presented here may play an interesting role in the statistics and thermodynamic of nonextensive systems recently discussed in the literature[10, 11].

Acknowledgments: This work was partially supported by Pronex/FINEP (No. 41.96.0908.00), Conselho Nacional de Desenvolvimento Científico e Tec- nológico - CNPq and CAPES (Brazilian Research Agencies), and by CONICET (Argentina Research Agency).

\section{References}

[1] P. T. Landsberg, Thermodynamics and Statistical Mechanics (Dover, New York, 1990), pp. 189-191.

[2] R. K. Pathria, Statistical Mechanics (Pergamon Press, New York, 1972), pp. 73-74.

[3] R. C. Tolman, The principles of Statistical Mechanics (Dover, New York, 1979), pp. 93.

[4] S. R. A. Salinas, Introduçâo à Física Estatística (Editora da Universidade de São Paulo, São Paulo, 1997), pp. 143.

[5] L. C. Woods, Kinetic Theory of Plasmas and Magnetoplasmas (Oxford University Press, Oxford, 1993), pp. 18.

[6] F. Reif, Fundamentals of Statistical and Thermal Physics (McGraw-hill, New York, 1965), pp. 248-250.

[7] L. E. Turner Jr., Generalized Classical Equipartition Theorem, Amer. J. Phys. 44, 104 (1976).

[8] F. S. Crawford, Elementary Derivation of the Law of Equipartition of Energy, Amer. J. Phys. 55, 180 (1987).

[9] P. T. Landsberg, Equipartition for a Relativistic Gas, Amer. J. Phys. 60, 561 (1992).

[10] C. Tsallis, Levy Distributions, Physics World 10, 42 (July 1997); A.R. Plastino and C. Anteneodo, A Dynamical Thermostatting Approach to Non-extensive Canonical Ensembles, Ann. Phys. 255, 250 (1997).

[11] R. Silva, A. R. Plastino and J. A. S. Lima, $A$ Maxwellian Path to the $q$-Nonextensive Velocity Distribution, Phys. Lett. A 249, 401 (1998).

[12] A. Lavagno, G. Kaniadakis, M. Rego-Monteiro, P. Quarati and C. Tsallis, Nonextensive Thermostatistical Approach to the Peculiar Velocity Function of Galaxy Clusters, Astrop. Lett. and Comm., 35, 449 (1998).

[13] I. S. Gradshteyn and I. M. Ryzhik, Table of Integrals, Series and Products (Academic Press, Orlando, Florida, 1980), pp. 284. 